\title{
Preparation and Properties of Cellulose Nanofibril- graphene Nanosheets/Polyaniline Composite Conductive Aerogels
}

\author{
Hui Zhang, ${ }^{\mathrm{a}}$ Tianyan Jiang, ${ }^{\mathrm{a}}$ Xinghua He, ${ }^{\mathrm{a}}$ Tiantian Chen, ${ }^{\mathrm{a}} \mathrm{Li}$ Fan, ${ }^{\mathrm{a}}$ Mengxi Gao, ${ }^{\mathrm{a}}$ and \\ Pengtao Liu ${ }^{\mathrm{a}, \mathrm{b}, *}$
}

\begin{abstract}
Polyaniline (PANI) is a conductive polymer that allows cellulose aerogels to achieve high electrical conductivity. However, aerogels containing PANI alone display a low mechanical stability. Graphene nanosheets (GNS) display high conductivity and mechanical strength but are prone to agglomeration, hindering their electroactive sites. To avoid shortcomings of the individual components, a composite aerogel was prepared via addition of graphene nanosheets (GNS) and PANI to a suspension of cellulose nanofibril (CNF). Transmission electron microscopy and scanning electron microscopy were used to analyze the structural morphology of the CNF/GNS/PANI aerogel. The electrochemical properties were analyzed using a four-probe conductivity meter, cyclic voltammetry, galvanostatic charge-discharge tests, and electrochemical impedance spectroscopy. A 2:2:1 ratio of CNF/GNS/PANI provided optimal structural and electrochemical results. Incorporation of PANI through in-situ polymerization for $6 \mathrm{~h}$ resulted in uniform mixing of the three components. The CNF/GNS/PANI composite aerogel displayed a high electrical conductivity with a specific capacitance of $375 \mathrm{Fg}^{-1}$ at a current density of $0.2 \mathrm{Ag}^{-1}$. As a base binder and dispersant, CNF made use of PANI as a conductive medium and of GNS as a conductive reinforcing medium to form a flexible nanocellulose composite conductive material with increased stability and improved performance.
\end{abstract}

Keywords: Cellulose; Polyaniline; Graphene; Aerogels; Conductive

Contact information: a: Tianjin Key Laboratory of Pulp \& Paper, Tianjin University of Science and Technology, Tianjin 300457, China; b: State Key Laboratory of Biobased Material and Green

Papermaking, Qilu University of Technology, Shandong Academy of Sciences, Jinan 250353,

China; *Corresponding author: pengtaoliu@tust.edu.cn

\section{INTRODUCTION}

Cellulose aerogels are a type of environmentally friendly material. They combine the advantages of cellulose regeneration, degradability, thermal properties, and chemical stability with the low density, high porosity, and toughness of aerogels (Fischer et al. 2006; Hoepfner et al. 2008). With the increased understanding of cellulose aerogels through research and the intensification of the global energy crisis, research on the use of cellulose aerogels has gradually increased. In recent years, cellulose aerogel-based materials with a highly permeable nanoporous three-dimensional network structure have shown significant functionality in the fields of photoelectric sensors and biomedicine (De France et al. 2017).

There are two main ways for the cellulose aerogels to achieve electrical conductivity: one uses carbonization (thermal cracking) treatment, which results in the formation of cellulose carbon aerogels, and the other uses composite conductive materials 
such as graphene and conductive polymers (Wan et al. 2018, 2014). Metal oxides, conductive polymers, and carbon-containing materials have become the electrode materials commonly produced for biomedical applications (Wu et al. 2014; Yang et al. 2014). However, each electrode material has unique advantages and disadvantages in supercapacitor applications. Some provide a longer life cycle while at the same time having a relatively low specific capacitance (Frackowiak 2007). In contrast, conductive polymer materials with higher specific capacitance due to redox properties have a relatively short life cycle and low mechanical stability (Hu et al. 2006; Zhang et al. 2011). Metal oxides often have limited applicability due to their high resistance, high cost, and toxicity (Wan 2008).

In recent years, conductive polymers have received particular attention in the field of composites because of their reversible doping/dedoping properties, highly conjugated polymer chains, and controlled conduction mechanisms (Fan et al. 2007; Bhadra et al. 2009). These unique properties play an important role in technical aspects of materials science. Among various conductive polymers, polyaniline (PANI) is one of the most studied because it is easy to synthesize, provides a stable environment, provides good conductivity, and can achieve unconventional rapid transition of conduction/insulation during doping/de-doping (Wang et al. 2009a). However, it undergoes expansion and contraction during doping/de-doping, which limits its cycle life, resulting in mechanical shedding of the electrode, a decline of electrochemical properties, and limitations in practical applications (Xu et al. 2012).

In contrast, research has found that graphene is an excellent candidate electrode material. Graphene is a two-dimensional nanomaterial composed of a carbon atoms consisting of overlapping sp2 hybrid orbital forming a hexagonal honeycomb lattice. It has extremely high thermal conductivity, a high specific surface area, high mechanical strength, and excellent electrical and optical properties (Novoselov et al. 2004). However, graphene nanosheets (GNS) are prone to agglomeration, hindering their electroactive sites. Therefore, pure graphene electrodes do not exhibit the desired capacitance (Wan et al. 2016).

To avoid the shortcomings of each single material in displaying both a good mechanical stability as well as excellent electrochemical properties, researchers are moving towards high-performance composite materials (Lee et al. 2008). A combination of PANI and graphene has been used to form a novel composite material by several conventional methods, such as in-situ polymerization or physical-mechanical mixing, which have been extensively studied to prepare high-performance materials (Esfandiar et al. 2011). Zhang et al. prepared a graphene/PANI nanofiber composite as an electrode with an electrochemical capacitance of $480 \mathrm{Fg}^{-1}$ (Zhang et al. 2010). Xu et al. (2010) prepared PANI/graphene oxide (GO) nanosheets as electrodes. Electrochemical studies have shown that PANI and GO have synergistic effects on the stability compared with single components, with nanocomposites reaching an electrochemical capacitance of $555 \mathrm{Fg}^{-1}$. Zheng et al. (2015) used cellulose nanofibril/reduced graphene oxide/carbon nanotube hybrid aerogels as electrodes, and the resulting flexible supercapacitors exhibited high specific capacitance (i.e., during discharge $252 \mathrm{~F} \mathrm{~g} \mathrm{~g}^{-1}$ at current density $0.5 \mathrm{Ag}^{-1}$ ) and significant cycle stability (that is, after 1000 charge-discharge cycles at a current density of $1 \mathrm{Ag}^{-1}$, more than $99.5 \%$ of the capacitance). Wang et al. (2009b) prepared a flexible graphene/PANI composite by in-situ electro polymerization on graphene paper. The results showed a tensile strength of $12.6 \mathrm{MPa}$ coupled with an electrochemical capacitance of 233 $\mathrm{Fg}^{-1}$. Therefore, its performance is better than many other flexible carbon-based electrodes 
currently available. Mao et al. (2011) prepared composite materials by in-situ polymerization of aniline under acidic conditions. Surfactants were added during the experiment to ensure the dispersion of the graphene in the aqueous phase, and the morphology and electrochemical properties of the two components were well maintained. The composite material obtained a high specific capacitance of $526 \mathrm{Fg}^{-1}$ at a current density of $0.2 \mathrm{Ag}^{-1}$, with good mechanical stability.

In this study, the authors first prepared nanofibrillated cellulose (or cellulose nanofibril, CNF, according to the ISO classification) and then added a mixture of surfactants and GNS to reduce agglomeration. The uniform dispersion of CNF can help to maintain the separation of adjacent GNS. the optimal ratio of CNF and GNS was first explored, and then the ability of two methods was compared (physical mixing and in situ polymerization of PANI) to obtain a layered porous network of mixed aerogel. A layered porous frame provides a large specific surface area and a rich pore structure, creating excellent ion accessibility and transportation potential, leading to a higher power performance (Fernández-Merino et al. 2010). The results show that the in-situ polymerization method allows a more uniform mixing of the three components. Electrochemical performance studies indicate that a CNF:GNS:PANI (2:2:1) composite aerogel has high specific capacitance and structural stability, which results in great advantages in the field of electrode materials for future energy storage systems.

\section{EXPERIMENTAL}

\section{Materials}

Bleached hardwood dissolving pulp (Asia Symbol Pulp and Paper Co. Ltd, Rizhao, China), the graphene nanosheets (Tokyo chemical industry Co. Ltd, Tokyo, Japan), hydrochloric acid ( $\mathrm{HCl}, 37 \%)$ (Binhua Group Co. Ltd, Binzhou, China), aniline (An), ammonium persulfate (APS), sodium dodecyl sulfate (SDS) (Shanghai Macklin Biochemical Co. Ltd, Shanghai, China), and 2,2,6,6-tetramethylpiperidin-1-oxyl (TEMPO) (Tokyo chemical industry Co. Ltd, Tokyo, Japan) were used in this work. All chemicals were analytical grade and deionized water was used throughout.

\section{Preparation and dispersion of $C N F$}

The raw fiber material was subjected to a dehydration and beating pretreatment, and then the product was placed in a $\mathrm{Na}_{2} \mathrm{CO}_{3} / \mathrm{NaHCO}_{3}$ buffer solution, followed by oxidization in a TEMPO/NaClO/NaBr system. The oxidized cellulose was dialyzed until the $\mathrm{pH}$ was neutral. Finally, CNF was obtained by high-pressure homogenization (Saito et al. 2007; Besbes et al. 2011).

\section{Preparation of CNF/GNS aerogels}

A reaction mixture was prepared containing solid graphene and $\mathrm{CNF}$ at various mass ratios $(20 \%, 30 \%, 40 \%, 50 \%$, and $60 \%$ graphene). The suspension was stirred with a magnetic stirrer for $2 \mathrm{~h}$, and then ultrasonicated for $1 \mathrm{~h}$ using an ultrasonic wave cell breaker at $600 \mathrm{~W}$. Next, the mixture was frozen in liquid nitrogen, and finally freeze-dried to obtain a CNF/GNS composite aerogel. The composite electrode sheet was obtained by tableting the aerogel under a pressure of $0.1 \mathrm{MPa}$. 


\section{Preparation of CNF/GNS/PANI Aerogels}

Physical mechanical mixing method

The CNF was prepared as a suspension with a concentration of $1 \%(\mathrm{w} / \mathrm{v})$. The GNS and SDS were placed into a suspension that was uniformly mixed by ultrasonication for 2 $\mathrm{h}$. The two suspensions were mixed, stirred with a magnetic stirrer for $2 \mathrm{~h}$, and then PANI was gradually added to the suspension in small portions so that the final mass ratio in the mixed system was CNF:PANI:GNS $=2: 1: 2$. The composite electrode sheet was obtained after freezing in liquid nitrogen, freeze drying for $48 \mathrm{~h}$, and tableting under a pressure of $0.1 \mathrm{MPa}$.

\section{In situ polymerization method}

As illustrated in Fig. 1, according to the above method, CNF and GNS were mixed and dispersed at a mass ratio of 1:1, uniformly mixed by stirring on a magnetic stirrer for $2 \mathrm{~h}$. Next, $10 \mathrm{~mL}$ of $\mathrm{HCl}$ and $25 \mathrm{mg}$ of aniline were added to the mixture, and then magnetic stirring was continued for $1 \mathrm{~h}$. Subsequently, $60 \mathrm{mg}$ of ammonium persulfate was added to $50 \mathrm{~mL}$ of $0.5 \mathrm{~mol} / \mathrm{L} \mathrm{HCl}$ solution, and then it was gradually added dropwise to the above mixture. The polymerization was allowed to proceed at $0{ }^{\circ} \mathrm{C}$ for $2 \mathrm{~h}, 3 \mathrm{~h}, 4 \mathrm{~h}, 6 \mathrm{~h}$, or $8 \mathrm{~h}$ after which the mixture was filtered under vacuum, washed, pre-frozen with liquid nitrogen, and then dried in a freeze-dried dryer for $48 \mathrm{~h}$ and pressed at a pressure of 0.1 MPa to obtain a composite electrode sheet.

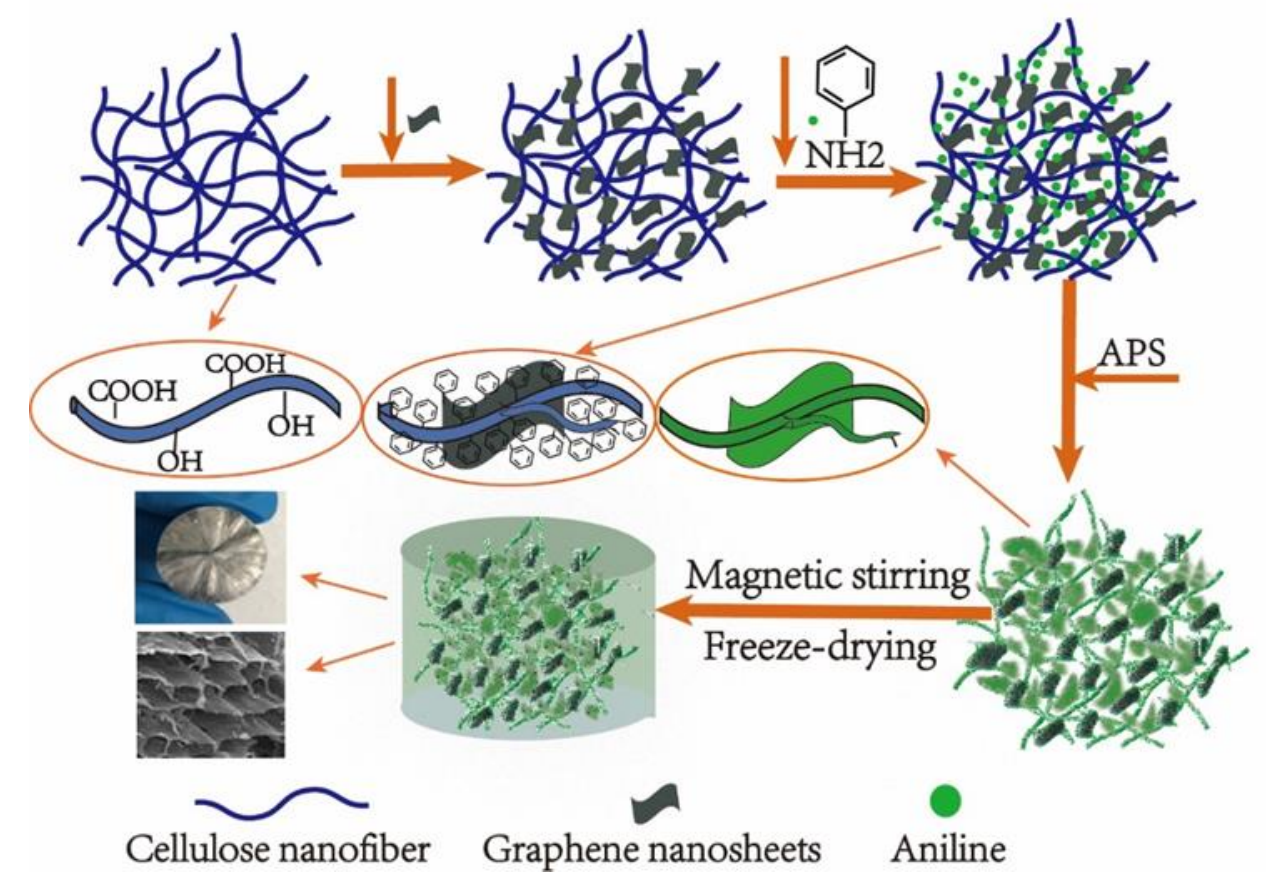

Fig. 1. Preparation of CNF/GNS/PANI composite aerogels by the in situ polymerization method

\section{Methods}

Characterization of CNF/GNS/PANI composite aerogels

The structure and morphology of the of CNF/GNS/PANI composite aerogels were characterized by transmission electron microscopy (TEM) (JEOL-2010; JEOL Japan Electronics Co. Ltd, Tokyo, Japan), energy dispersive spectrometer (EDS) (GeminiSEM300; Carl Zeiss AG, Berlin, Germany), electronic universal tester (TMC4503; 
Jinan hengsi Shanda Instrument Co. Ltd, Jinan, China), Fourier transform infrared spectrometer (FT-IR) (IRTracer-10; Shimadzu Co. Ltd, Tokyo, Japan), specific surface area pore size analyzer (Autosorb-iQ-XR; Quantachrome, USA), and scanning electron microscopy (SEM) (JSM-IT300LV; JEOL Japan Electronics Co. Ltd, Tokyo, Japan). Electrochemical characterization was performed using a four-probe conductivity meter (RTS-8; Suzhou lattice Electronics Co. Ltd, Suzhou, China), electrochemical workstation (CHI650E; Shanghai Chenhua Instrument Co. Ltd, Shanghai, China) for cyclic voltammetry (CV), galvanostatic constant current charge-discharge (GCD) technology, and electrochemical impedance spectroscopy (EIS). The potential range measured by $\mathrm{CV}$ was -0.2 to $0.8 \mathrm{~V}$. The GCD voltage window ranged from $0 \mathrm{~V}$ to $0.8 \mathrm{~V}$, the segment was set to 4 , and the current density range was set from $0.2 \mathrm{~A} / \mathrm{g}$ to $1 \mathrm{~A} / \mathrm{g}$. The EIS initial potential was set to open circuit voltage, the frequency range was set from $0.01 \mathrm{~Hz}$ to $100000 \mathrm{~Hz}$. The three-electrode setting was used, where the composite material was used as the working electrode, the platinum electrode was used as the counter electrode, the calomel electrode was used as the reference electrode, and a $1.0 \mathrm{M} \mathrm{H}_{2} \mathrm{SO}_{4}$ solution was used as the electrolyte.

\section{RESULTS AND DISCUSSION}

\section{Morphological and Structural Characterization}

Figure 2 shows a transmission electron micrograph of CNF. The $\mathrm{Na}_{2} \mathrm{CO}_{3} / \mathrm{NaHCO}_{3}$ buffer system provided a relatively stable $\mathrm{pH}$ environment for the oxidation reaction, thereby improving the selectivity of the TEMPO-dependent catalytic oxidation. There was no obvious agglomeration of $\mathrm{CNF}$, the size and distribution of the fibers were relatively uniform, with a good dispersion.
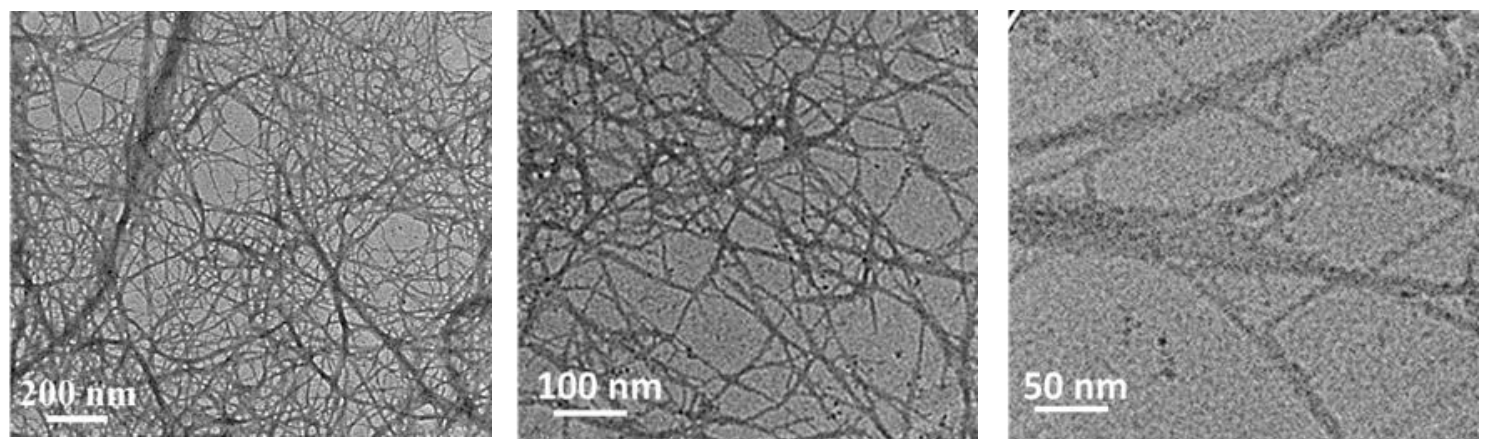

Fig. 2. TEM of CNF showing no obvious agglomeration, uniform fiber distribution, and good dispersion

Figure 3 shows the effect of SDS on the dispersion of graphene. The SDS acted as a surfactant for GNS that still allowed GNS to be evenly dispersed in water after $24 \mathrm{~h}$ (vial b). In the absence of SDS, the GNS solution was completely stratified after $6 \mathrm{~h}$ (vial a). Thus, SDS provided a stable environment for the three composites and reduced the agglomeration of GNS.

In the composite system, the pore structure is associated with the graphene content, and the optimum ratio of GNS:CNF was experimentally explored. Figure 4 shows the pore structure when GNS:CNF was 1:1 (panel a and b), and the state of the structure when GNS:CNF was 3:2 (panel $\mathrm{c}$ and d). It can be seen from Fig. 4 that an increase in the 
GNS:CNF ratio from 1:1 to $3: 2$ led to a loosening of the structure, characterized by suboptimal pore formation and mechanical strength. Therefore, the optimal ratio of GNS:CNF for the structure of the composite aerogel is $1: 1$.

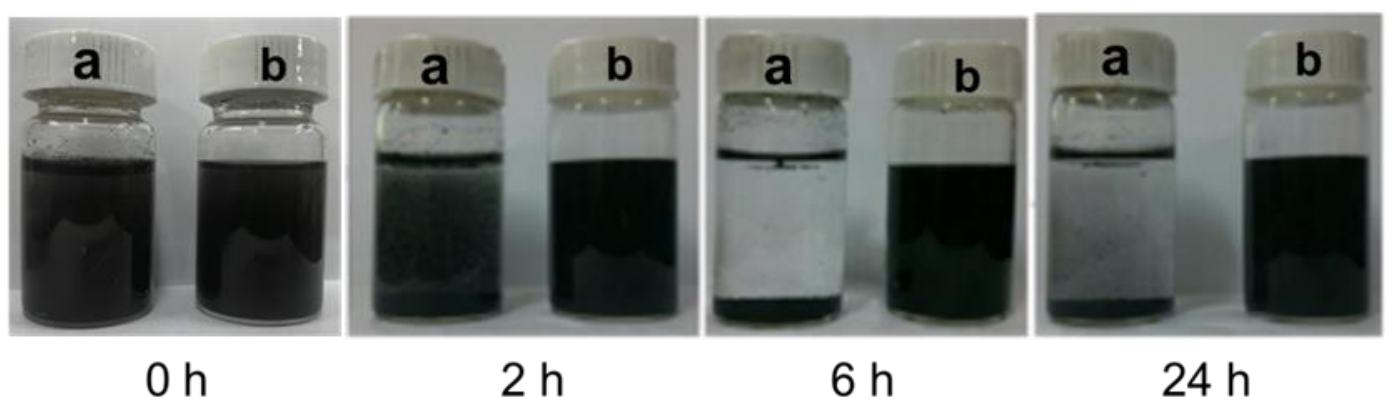

Fig. 3. Effect of SDS on the distribution of GNS (a: graphene without SDS; b: graphene with SDS)
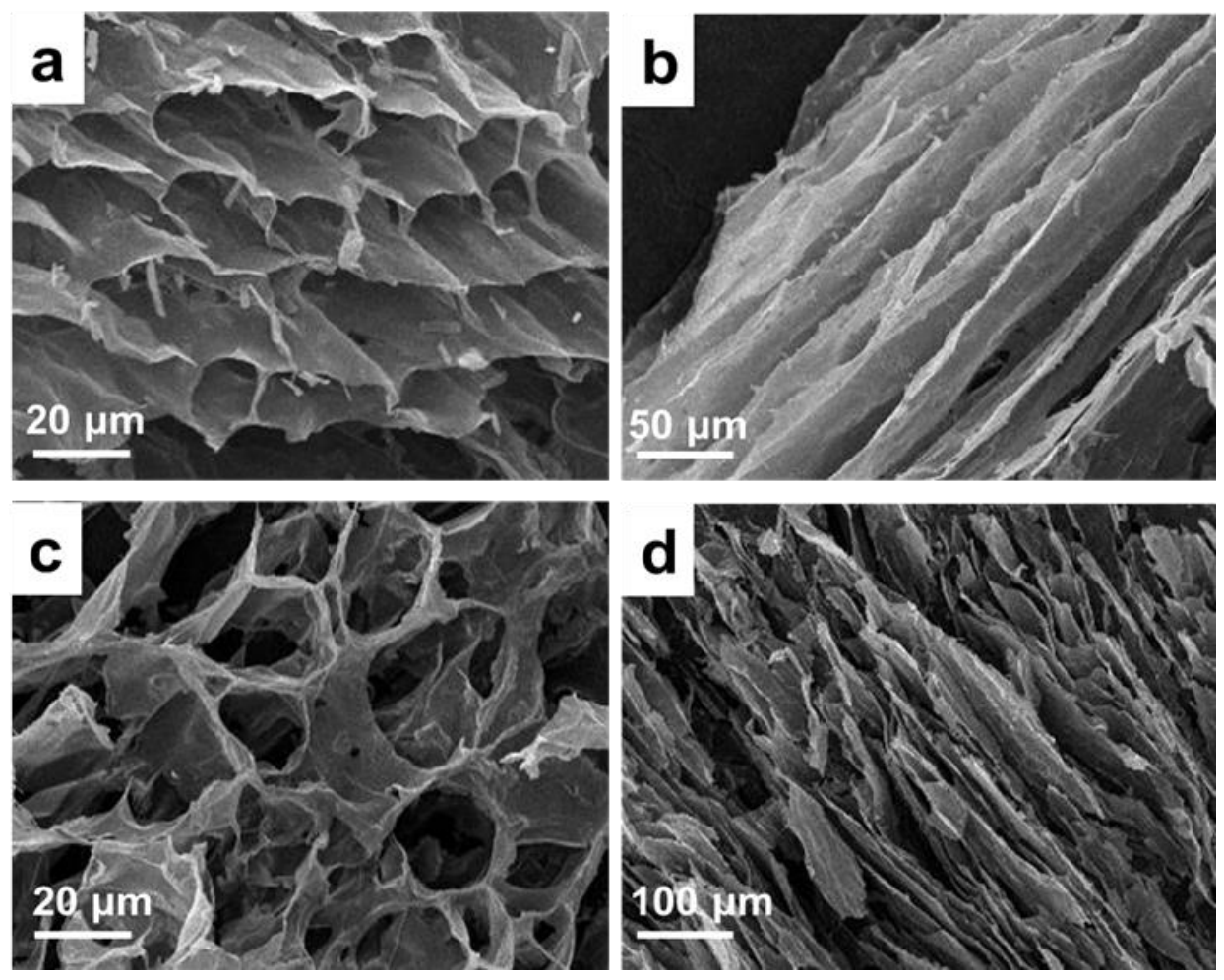

Fig. 4. SEM image of CNF/GNS composite aerogel structure (a) and (b) where GNS: CNF = 1:1; (c) and (d) GNS: CNF = 3:2

In-situ polymerization combines aniline doping with CNF and GNS to form polyaniline. Figure 5 shows the FT-IR spectra of the CNF, GNS, PANI, and CNF/GNS/PANI composites. For CNF, three characteristic peaks at 1061, 1615 and 2903 $\mathrm{cm}^{-1}$ indicate the presence of a hydroxyl group and a carboxyl group. For GNS, three characteristic peaks at 1176,1400 , and $1635 \mathrm{~cm}^{-1}$ represent the bending vibration peak of $-\mathrm{OH}$, the stretching vibration peak of the secondary alcohol, and the deformation vibration peak of the benzene ring. For pure PANI, the peaks at 1592 and $1498 \mathrm{~cm}^{-1}$ correspond to the characteristic $\mathrm{C}-\mathrm{C}$ extensions of the oxime and benzene rings, respectively, and the 
peaks at 1304 and $1145 \mathrm{~cm}^{-1}$ are related to the $\mathrm{C}-\mathrm{N}$ and $\mathrm{C}=\mathrm{N}$ stretching vibrations. The prepared CNF/GNS/PANI complex has obvious characteristic peaks of GNS and PANI, and the peak intensity is weakened, indicating that the interaction between the three is tight.

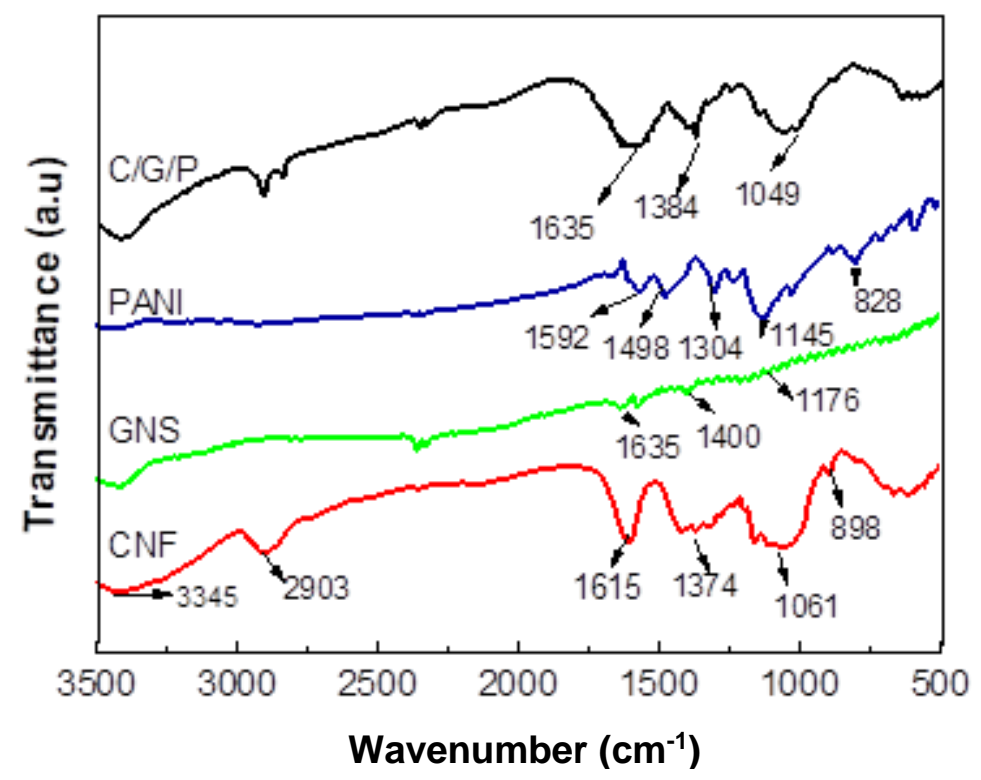

Fig. 5. FT-IR spectrum of CNF, GNS, PANI and in-situ polymerized composite aerogel
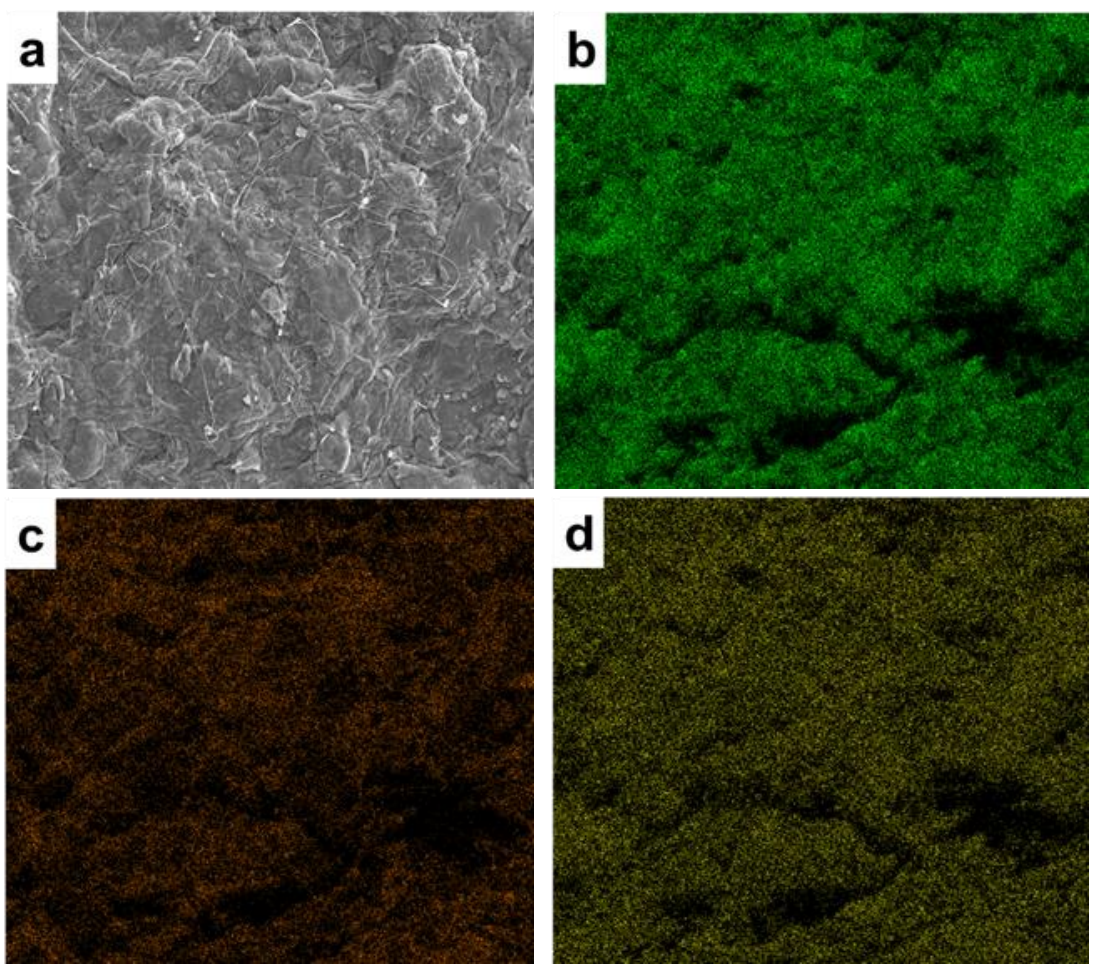

Fig. 6. The SEM image of (a) In-situ polymerized composite aerogel; the element mapping images of (b) $\mathrm{C}$ atom, (c) $\mathrm{O}$ atom and (d) $\mathrm{N}$ atom

The analysis of EDS showed that the distribution points of $\mathrm{C}, \mathrm{O}$, and $\mathrm{N}$ elements in the composite aerogel prepared by in situ polymerization were very close, indicating that 
CNF, GNS, and PANI were distributed uniformly in the composite. Based on the analysis results of the above infrared spectrum, it is shown that the PANI formed by in situ polymerization is tightly combined with CNF and GNS.

Composite aerogels prepared by two different methods (i.e., mechanical mixing vs. in-situ polymerization) also differ in morphology. Figure 7 shows agglomeration of PANI on the surface (red ellipse in panel b) and on the inside (red ellipse in panel a) of the aerogel prepared by mechanical mixing, displaying a loose structure. In contrast, the aerogel prepared by the in-situ polymerization method had a regular pore structure, and PANI agglomeration rarely occurred. Thus, the aerogel obtained by the in-situ polymerization method was of superior quality.
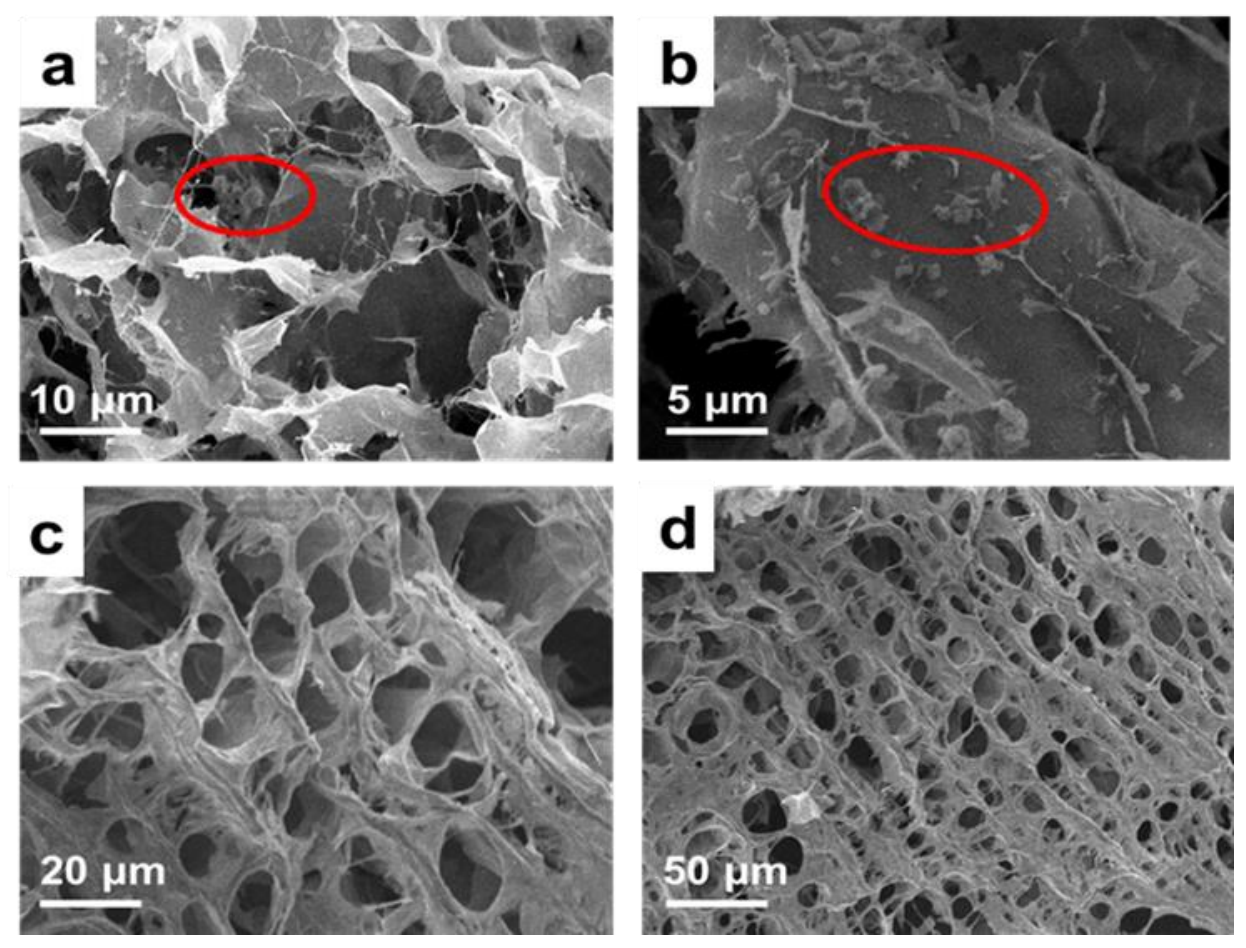

Fig. 7. SEM images of composite aerogel prepared by mechanical mixing (a) and (b) or by in-situ polymerization (c) and (d)

The isotherms of the prepared composite aerogels belong to type IV, which is indicative of a mesoporous material, and the pore size was mostly between 5 and $20 \mathrm{~nm}$. The aerogel prepared by in-situ polymerization had a larger adsorption capacity and a more uniform pore size distribution, mainly between 2 and $10 \mathrm{~nm}$. The specific surface area of the mechanically-mixed composite aerogel was $65.1 \mathrm{~m}^{2} / \mathrm{g}$, while the material resulting from in-situ polymerization reached $134.2 \mathrm{~m}^{2} / \mathrm{g}$.

When GNS was added at $50 \%$, it provided better compressive properties. The compressive stress could reach $1.9 \mathrm{MPa}$, indicating a high mechanical strength, which is consistent with the results of SEM image analysis. Compared with the mechanical mixing method, the in-situ polymerization method has obvious advantages. The compression strain can reach $2.35 \mathrm{MPa}$, which has higher mechanical strength, which is consistent with the above analysis. 

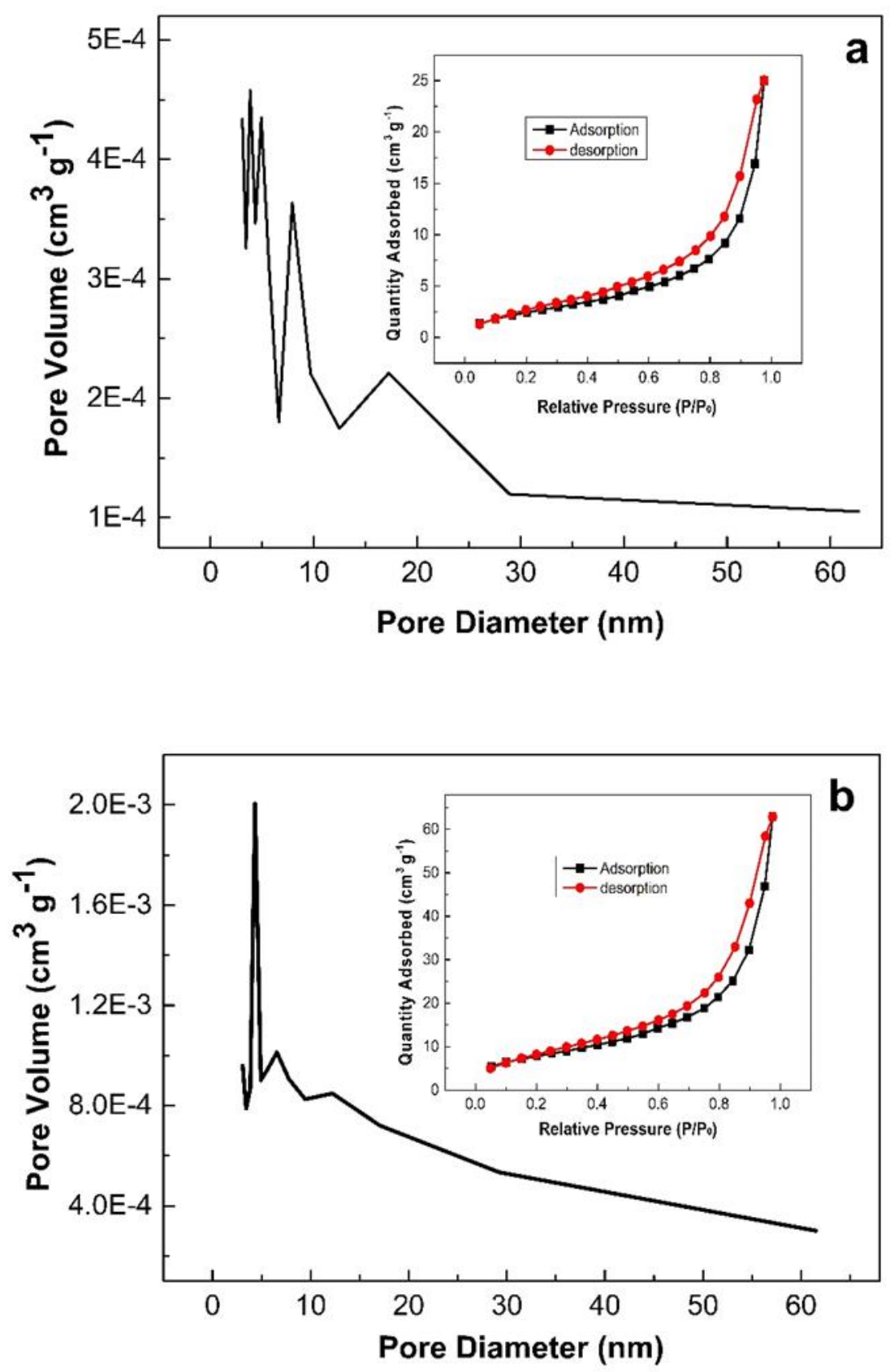

Fig. 8. $\mathrm{N}_{2}$ adsorption-desorption isotherm and pore diameter analysis of composite aerogels (a) mechanical mixing method; (b) in situ polymerization method 

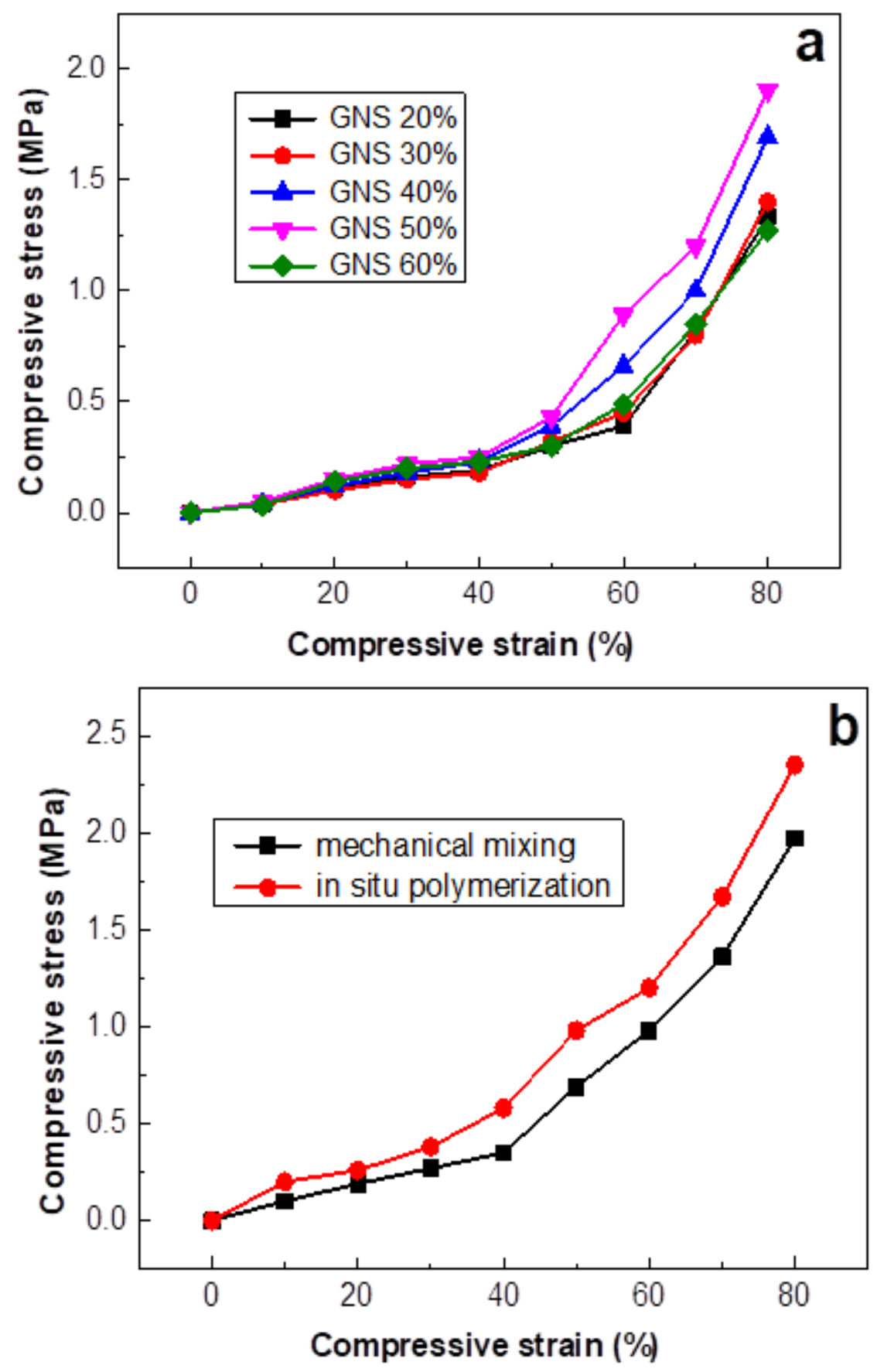

Fig. 9. Stress-strain curve of the composite aerogel (a) different GNS content; (b) different preparation methods

\section{Electrochemical Characterization}

The conductivity of the electrode sheets was measured using a four-probe conductivity meter. The measurement points were $(0,0),(0,1),(0,-1),(1,0)$, and $(-1,0)$, and the average conductivity of each electrode was obtained. As shown in Fig. 10, as the percentage of GNS increased, the average conductivity of the electrode also increased. When the GNS content exceeded 50\%, the electrical conductivity still increased (to 7.21 $\mathrm{S} / \mathrm{cm}$ at $60 \% \mathrm{GNS}$ ), but the surface structure of the electrode was loosened and the GNS 
fell off, resulting in the loss of active material. Therefore, the authors found that when the GNS:CNF ratio was 1:1, the dispersion and mechanical properties were optimal.

Figure 11 shows that as the in-situ polymerization time of PANI increased, the average conductivity of the electrode also gradually increased. When the polymerization time was $6 \mathrm{~h}$, the conductivity reached a maximum of $11.78 \mathrm{~S} / \mathrm{cm}$. When the polymerization time exceeded $6 \mathrm{~h}$, the conductivity decreased. In an oxidizing environment, the excessive polymerization time of aniline caused oxidative degradation of the formed molecular chain, thereby reducing its electrical conductivity.

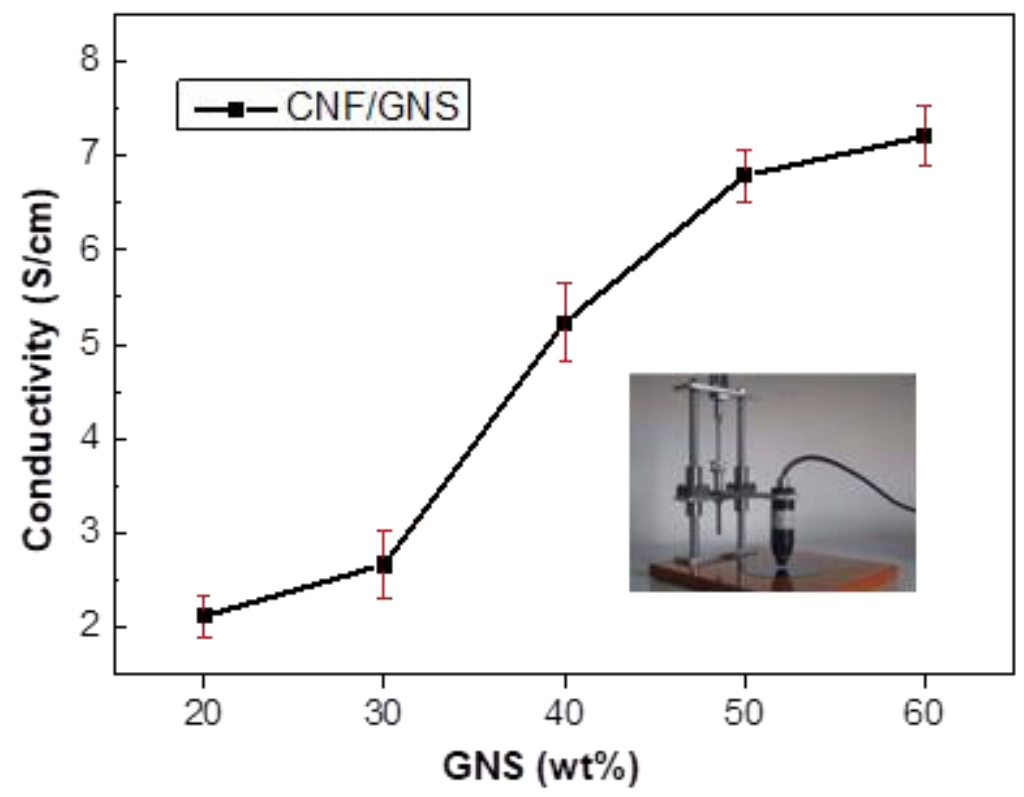

Fig. 10. Conductivity test of composite electrode with different CNF/GNS ratios

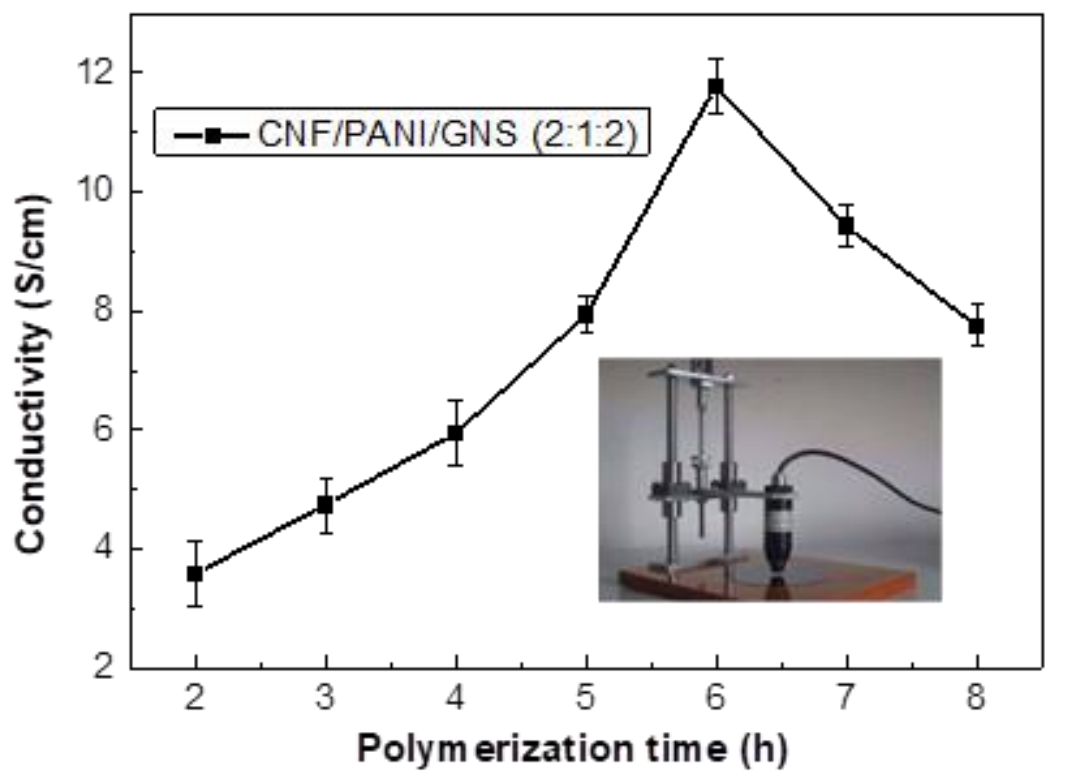

Fig. 11. Effect of different in-situ polymerization times of PANI on the conductivity of CNF/PANI/GNS (2:1:2) aerogels 
Based on the cyclic voltammetry curve and the mass of the active material on the electrode, the specific capacitance of the electrode material can be calculated by Eq. 1,

$$
C_{c v}=\frac{\int I d V}{v \Delta V m}
$$

where $C_{\mathrm{cv}}$ is the specific capacitance of composite materials $(\mathrm{F} / \mathrm{g}), I$ is the current (A), $V$ is the potential window $(\mathrm{V}), m$ is the mass of the active material on the electrode $(\mathrm{g})$, and $v$ is the canning speed $(\mathrm{mV} / \mathrm{s})$. Eq. 2,

The specific capacity of the composite material can also be calculated according to

$$
C_{c d}=\frac{I t}{m \Delta V}
$$

where $C_{\mathrm{cd}}$ is the specific capacity of the electrode material $(\mathrm{F} / \mathrm{g}), I$ is the current $(\mathrm{A}), t$ is the discharge time (s), $V$ is the voltage difference after IR drop (V), and $m$ is the active material on the electrode quality $(\mathrm{g})$.

Figure 12 shows that the cyclic volt-ampere curves of different scan rates displayed a rectangular shape, which means that the internal resistance of the composite electrode was small, and that the power characteristics were good. There was no obvious redox peak, which indicated that the composite electrode had good capacitance characteristics and reversibility. The specific capacitances of the composite electrode at the scan rates of 5, 10, 30, 50, and $100 \mathrm{mV} / \mathrm{s}$ were calculated using Eq. 1 to be 437.5, 325.6, 241.8, 215.2, and $183.7 \mathrm{~F} / \mathrm{g}$, respectively.

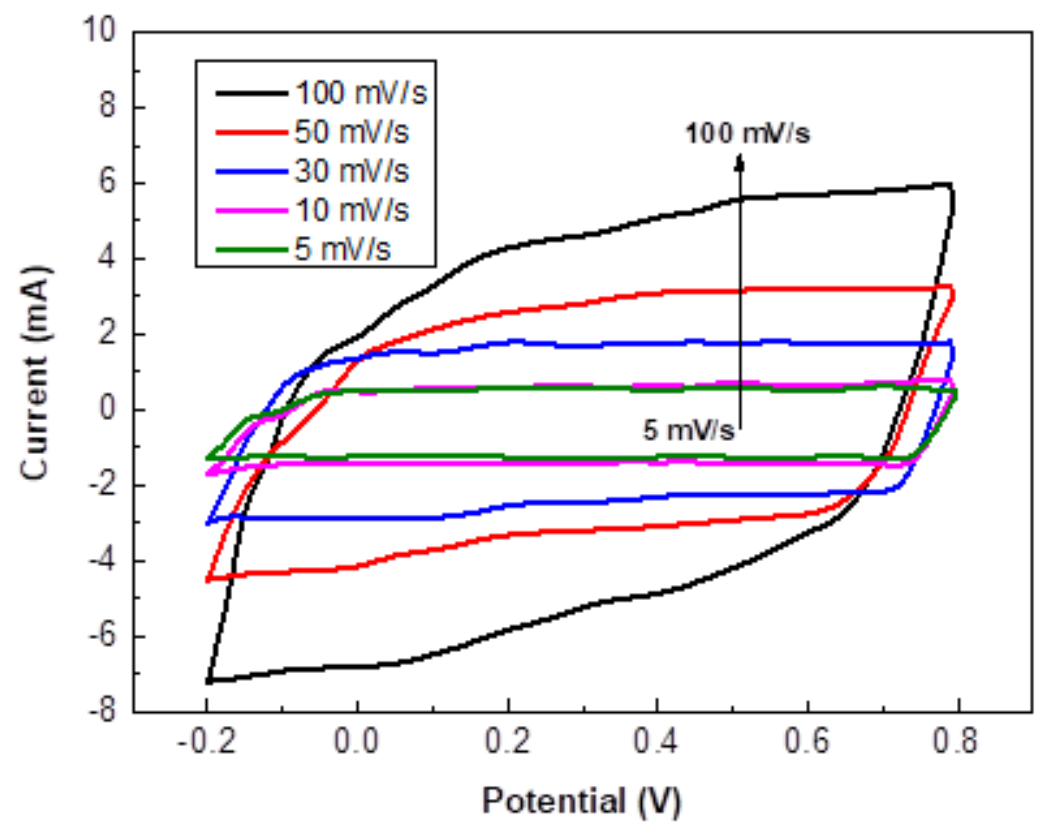

Fig. 12. Effect of scan rates on the cyclic volt-ampere curves of the composite aerogel electrode

The rectangular shape indicated small internal resistance of the composite electrode. Absence of redox peak indicated that the composite electrode had good capacitance characteristics and reversibility. 
The charge-discharge curves exhibited a triangular characteristic at different current densities (Fig. 13), which indicated that the voltage of the electrode had a linear relationship with the charge and discharge time and exhibited good charge and discharge performance. There was a small pressure drop at the beginning of the discharge, indicating that the internal resistance of the composite electrode was small. The specific capacitances of the composite electrodes at current densities of $0.2,0.3,0.5$, and $1 \mathrm{~A} / \mathrm{g}$ were calculated by Eq. 2 to be 375, 283, 208, and $169 \mathrm{~F} / \mathrm{g}$, respectively.

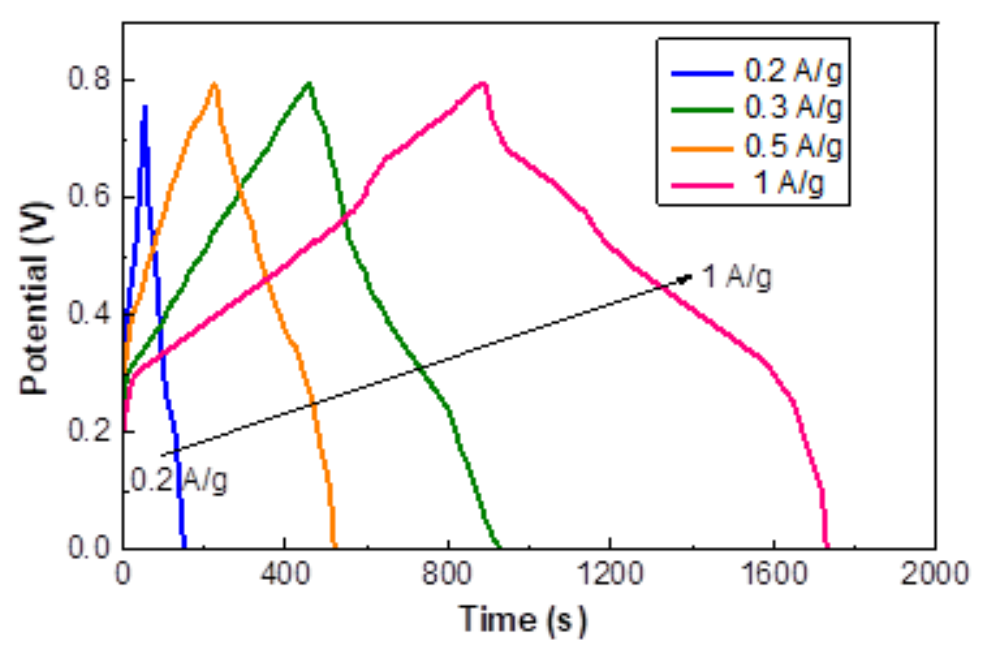

Fig. 13. Galvanostatic charge-discharge (GCD) of composite aerogel electrode at various current densities

The EIS initial potential was set to open circuit voltage, and the frequency range was set from $0.01 \mathrm{~Hz}$ to $100000 \mathrm{~Hz}$. The CNF/GNS/PANI aerogel was the working electrode, the platinum electrode was the counter electrode, and the calomel electrode was the reference electrode. $1.0 \mathrm{M} \mathrm{H}_{2} \mathrm{SO}_{4}$ was the electrolyte. Above $80 \mathrm{ohm}$ the curve was almost perpendicular to the $Z^{\prime}$ axis, indicating that the composite electrode had excellent capacitance properties. Figure 14 shows that as the $Z^{\prime}$ axis increased continuously, the curve in the figure had three stages of changes. 


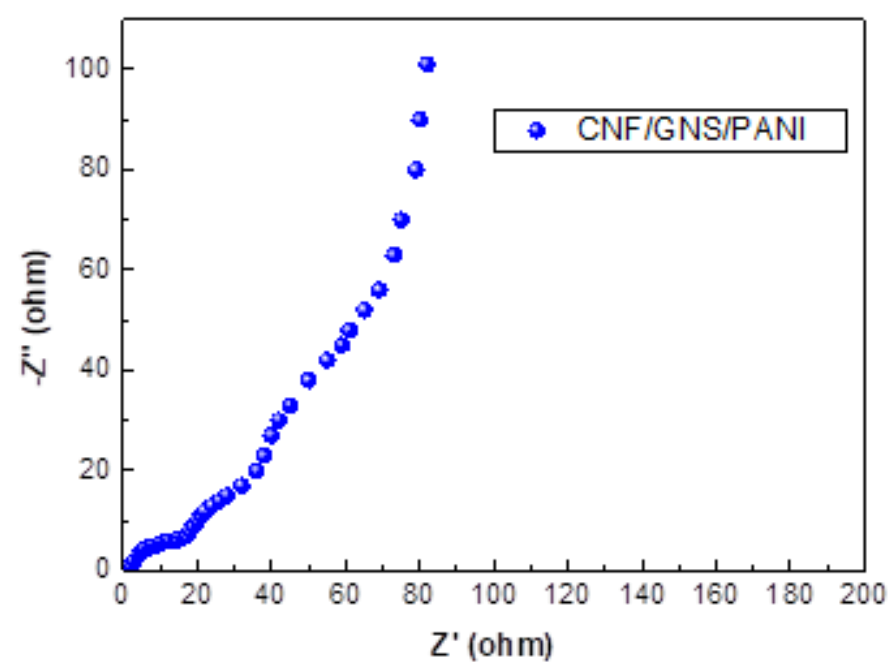

Fig. 14. Nyquist plot for the composite CNF/GNS/PANI aerogel electrode

The first segment was semicircular, indicating the high frequency region of the electrode. The second segment was a straight line with a slope of approximately $45^{\circ}$, corresponding to the intermediate frequency region. The third segment was a straight line approximately parallel to the $\mathrm{Z} "$ axis, corresponding to the low frequency region of the electrode. The internal resistance of the electrolyte was $15.8 \Omega$. A line almost perpendicular to the $\mathrm{Z}^{\prime}$ axis indicates that the composite electrode has excellent capacitance properties.

\section{CONCLUSIONS}

1. SEM images and pore size distributions analysis revealed that in situ polymerization of aniline caused polyaniline (PANI) to be uniformly dispersed on the inside and surface of aerogels comprised of nanofibrillated cellulose (CNF), graphene nanosheets (GNS) and PANI, retaining the original loose porous structure. Compared with the mechanical mixing method, it had higher compressive stress.

2. Tests on its different electrochemical properties showed that CNF/GNS/PANI composite electrodes had excellent electrical conductivity and were suitable as electrode materials.

3. Both GNS and CNF formed a three-dimensional network, which provided a stable basic support for PANI. In summary, the three composite CNF/GNS/PANI aerogels are excellent electrode materials that are ideal for supercapacitors.

\section{ACKNOWLEDGMENTS}

The financial support for this project was from the National Key Research and Development Plan (No. 2017YFB0307902), the National Nature Science Foundation of China (No. 21576213), the Scientific Research Project of Tianjin Municipal Education Commission (No. 2018KJ098), the Foundation of State Key Laboratory of Biobased Material \& Green Papermaking (No. KF201809), and the Young Teachers' Innovation Fund of Tianjin University of Science and Technology (No. 2017LG06). 


\section{REFERENCES CITED}

Besbes, I., Alila, S., and Boufi, S. (2011). "Nanofibrillated cellulose from tempo-oxidized eucalyptus fibres: Effect of the carboxyl content," Carbohyd. Polym. 84(3), 975-983. DOI: 10.1016/j.carbpol.2010.12.052

Bhadra, S., Khastgir, D., Singha, N. K., and Lee, J. H. (2009). "Progress in preparation, processing and applications of polyaniline," Prog. Polym. Sci. 34(8), 783-810. 10.1016/j.progpolymsci.2009.04.003

De France, K. J., Hoare, T., and Cranston, E. D. (2017). "Review of hydrogels and aerogels containing nanocellulose," Chem. Mater. 29(11), 4609-4631. DOI: 10.1021/acs.chemmater.7b00531

Esfandiar, A., Akhavan, O., and Irajizad, A. (2011). "Melatonin as a powerful bioantioxidant for reduction of graphene oxide," J. Mater. Chem. 21(29), 10907-10914. DOI: $10.1039 / \mathrm{C} 1 \mathrm{JM} 10151 \mathrm{~J}$

Fischer, F., Rigacci, A., Pirard, R., Berthon-Fabry, S., and Achard, P. (2006). "Cellulosebased aerogels," Polymer 47(22), 7636-7645. DOI: 10.1016/j.polymer.2006.09.004

Frackowiak, E. (2007). "Carbon materials for supercapacitor application," Phys. Chem. Chem. Phys. 9(15), 1774-1785. DOI: 10.1039/b618139m

Fan, L. Z., Hu, Y. S., Maier, J., Adelhelm, P., Smarsly, B., and Antonietti, M. (2007). "High electroactivity of polyaniline in supercapacitors by using a hierarchically porous carbon monolith as a support," Adv. Funct. Mater. 17(16), 3083-3087. 10.1002/adfm.200700518

Fernández-Merino, M. J., Guardia, L., Paredes, J. I., Villar-Rodil, S., Solís-Fernández, P., Martínez-Alonso, and Tascón, J. M. D. (2010). "Vitamin C is an ideal substitute for hydrazine in the reduction of graphene oxide suspensions," J. Phys. Chem. C 114(14), 6426-6432. DOI: 10.1021/jp100603h

Hoepfner, S., Ratke, L., and Milow, B. (2008). "Synthesis and characterisation of nanofibrillar cellulose aerogels," Cellulose 15(1), 121-129. DOI: 10.1007/s10570007-9146-8

Hu, C. C., Chang, K. H., Lin, M. C., and Wu, Y. T. (2006). "Design and tailoring of the nanotubular arrayed architecture of hydrous $\mathrm{RuO}_{2}$ for next generation supercapacitors," Nano Lett. 6(12), 2690-2695. DOI: 10.1021/nl061576a

Lee, C., Wei, X., Kysar, J. W., and Hone, J. (2008). "Measurement of the elastic properties and intrinsic strength of monolayer graphene," Science 321(5887), 385388. DOI: $10.1126 /$ science. 1157996

Mao, L., Zhang, K., Chan, H. S. O., and Wu, J. (2011). "Surfactant-stabilized graphene/polyaniline nanofiber composites for high performance supercapacitor electrode," J. Mater. Chem. 22(1), 80-85. DOI: 10.1039/C1JM12869H

Novoselov, K. S., Geim, A. K., Morozov, S. V., Jiang, D., Zhang, Y., Dubono, S. V., Grigorieva, I. V., and Frisov, A. A. (2004). "Electric field effect in atomically thin carbon films," Science 306(5696), 666-669. DOI: 10.1126/science.1102896

Saito, T., Kimura, S., Nishiyama, Y., and Isogai, A. (2007). "Cellulose nanofibers prepared by tempo-mediated oxidation of native cellulose," Biomacromolecules 8(8), 2485-2491. DOI: 10.1021/bm0703970

Wang, D., Huang, J., Lan, W., and Li, X. (2009a). "Neural network-based robust adaptive control of nonlinear systems with unmodeled dynamics," Math. Comput. Sim. 79(5), 1745-1753. DOI: 10.1016/j.matcom.2008.09.002 
Wang, D. W., Li, F., Zhao, J., Ren, W., Chen, Z. G., and Tan, J., Wu, Z. S., Gentle, I., Lu, G. Q., and Cheng, H. M. (2009b). "Fabrication of graphene/polyaniline composite paper via in situ anodic electropolymerization for high-performance flexible electrode," ACS Nano 3(7), 1745-1752. DOI: 10.1021/nn900297m

Wan, M. (2008). "A template-free method towards conducting polymer nanostructures," Adv. Mater. 20(15), 2926-2932. DOI: 10.1002/adma.200800466

Wan, C., Lu, Y., Jiao, Y., Jin, C., and Li, J. (2014). "Fabrication of hydrophobic, electrically conductive and flame-resistant carbon aerogels by pyrolysis of regenerated cellulose aerogels," Carbohyd. Polyme. 118C. DOI: 10.1016/j.carbpol.2014.11.010

Wan, C., and Li, J. (2016). "Graphene oxide/cellulose aerogels nanocomposite: Preparation, pyrolysis, and application for electromagnetic interference shielding," Carbohyd. Polym. S0144861716305732. DOI: 10.1016/j.carbpol.2016.05.051

Wan, C., Jiao, Y., Wei, S., Zhang, L. Y., and Wu, Y. Q. (2018). "Functional nanocomposites from sustainable regenerated cellulose aerogels: A review," Chem. Eng. J. 359, 459-475. DOI: 10.1016/j.cej.2018.11.115

Wu, W., Li, Y., Yang, L., Ma, Y., Pan, D., and Li, Y. (2014). "A facile one-pot preparation of dialdehyde starch reduced graphene oxide/polyaniline composite for supercapacitors," Electrochim. Acta 139, 117-126. DOI:

10.1016/j.electacta.2014.06.166

Xu, G., Wang, N., Wei, J., Lv, L., Zhang, J., Chen, Z., and Xu, Q. (2012). "Preparation of graphene oxide/polyaniline nanocomposite with assistance of supercritical carbon dioxide for supercapacitor electrodes," Ind. Eng. Chem. Res. 51(44), 14390-14398. DOI: 10.1021/ie301734f

Xu, J., Wang, K., Zu, S. Z., Han, B. H., and Wei, Z. (2010). "Hierarchical nanocomposites of polyaniline nanowire arrays on graphene oxide sheets with synergistic effect for energy storage," ACS Nano 4(9), 5019-5026. DOI: $10.1021 / \mathrm{nn} 1006539$

Yang, F., Xu, M., Bao, S. J., Wei, H., and Chai, H. (2014). "Self-assembled hierarchical graphene/polyaniline hybrid aerogels for electrochemical capacitive energy storage," Electrochim. Acta 137, 381-387. DOI: 10.1016/j.electacta.2014.06.017

Zheng, Q., Cai, Z., Ma, Z., and Gong, S. (2015). “Cellulose nanofibril/reduced graphene oxide/carbon nanotube hybrid aerogels for highly flexible and all-solid-state supercapacitors," ACS Appl Mater \& Inter 7(5), 3263-3271. DOI: $10.1021 / \mathrm{am} 507999 \mathrm{~s}$

Zhang, W. J. (2011). "A review of the electrochemical performance of alloy anodes for lithium-ion batteries," J. Power Sources 196(1), 13-24. DOI: 10.1016/j.jpowsour.2010.07.020

Zhang, K., Zhang, L. L., Zhao, X. S., and Wu, J. (2010). "Graphene/polyaniline nanofiber composites as supercapacitor electrodes," Chem. Mater. 22(4), 1392-1401. DOI: $10.1021 / \mathrm{cm} 902876 \mathrm{u}$

Article submitted: October 21, 2019; Peer review completed: December 8, 2019; Revised version received: January 20, 2020; Accepted: January 21, 2020; Published: January 27, 2020.

DOI: 10.15376/biores.15.1.1828-1843 